\title{
Solute-rich clusters as nucleation medium of small organic molecules: a case of p-nitrobenzoic acid
}

\author{
Shuyi Zong ${ }^{1}$, Jingkang Wang ${ }^{1}$, Xin Huang ${ }^{2}$, Yaoguang Feng ${ }^{1}$, Na Wang ${ }^{2}$, Ting Wang ${ }^{2}$, Qi \\ $\mathrm{Liu}^{1}$, and Hongxun $\mathrm{HaO}^{2}$ \\ ${ }^{1}$ Tianjin University \\ ${ }^{2}$ Tianjin university
}

March 8, 2022

\begin{abstract}
Solute-rich clusters, which generally exist stably in solution, are necessary intermediates for the formation of crystal nuclei. Studying the properties of these pre-nucleation species is very important for understanding and controlling the nucleation process of crystals. In this work, clusters as nucleation medium were studied using p-nitrobenzoic acid (PNBA) in acetonitrile as model system, and the observed PNBA clusters were characterized and analyzed by using DLS, NTA and 1H NMR. It was found that the clusters were thermodynamically stable and were spatially heterogeneous mixtures of PNBA monomers, PNBA homodimers and acetonitrile molecules. The existence of dimers is an important factor for the formation and stable existence of clusters. Clusters can be formed under the combined effect of monomer-dimer reaction and diffusion. The spatial distributions of monomer and dimer density at different concentrations were obtained by reaction-diffusion kinetics, and the different effects of filtration on clusters were theoretically explained.
\end{abstract}

\section{Hosted file}

manuscript.docx available at https://authorea.com/users/464015/articles/559011-solute-richclusters-as-nucleation-medium-of-small-organic-molecules-a-case-of-p-nitrobenzoic-acid 